\title{
Development and Simulation of a Control Strategy for a Two Finger Parallel Electric Gripper
}

\author{
Paige Gallant \\ Department of Mechanical Engineering \\ University of New Brunswick \\ Fredericton, NB, Canada \\ paige.gallant@unb.ca
}

\author{
Rickey Dubay \\ Department of Mechanical Engineering \\ University of New Brunswick \\ Fredericton, NB, Canada \\ dubayr@unb.ca
}

\begin{abstract}
Robotic gripping is a functionality that is becoming increasingly important with the growth and development of autonomous machinery and collaborative robots.The human grasp is a complex dynamic system and mimicking its function with a robotic gripper is a challenging task. This paper develops a strategy to control both position and force of a two-finger parallel gripper using cascading control schemes and a switching mechanism. The performance of different control schemes were compared in simulation to determine a preeminent and feasible process control that can be implemented on an experimental setup and eventually be used in industry.

Index Terms-model predictive control, robotic gripping, PD, PI, linear actuator
\end{abstract}

\section{INTRODUCTION}

Researchers are striving to develop more human-like robots, and a stepping stone towards this goal is to mimic the human grasp [1]. Many traditional robotic grippers are application specific with rigid fingers and singular force and position trajectories. This is especially the case with pneumatic grippers. Electric grippers are more accommodating to control schemes and can be easily controlled with feedback from a wide variety of sensors [2]. This work explores a proof of concept for an electric two-finger parallel gripper that is able to accommodate a variety of objects with different sizes and weights and accurately control the gripping forces in manner similar to humans. The desired outcome of this paper is to control both the position of the gripper fingers and the amount of force applied to an object being grasped. This requires separate control schemes for each and a switching mechanism that recognizes when the fingers have made contact with an object and will subsequently maintain a desired gripping force. This loosely mimics the way human use both kinesthetic and tactile feedback to pick up objects [3].

\section{DyNAmic Model}

The system that comprises the two-finger parallel electric gripper is made up of a brushed DC motor connected to a lead screw mechanism with one finger stationary and the other fixed to the lead screw table. A schematic is shown in Fig. 1. Plant models were developed for both the actuator force and the finger position. Both outputs were derived from the model of a DC motor.

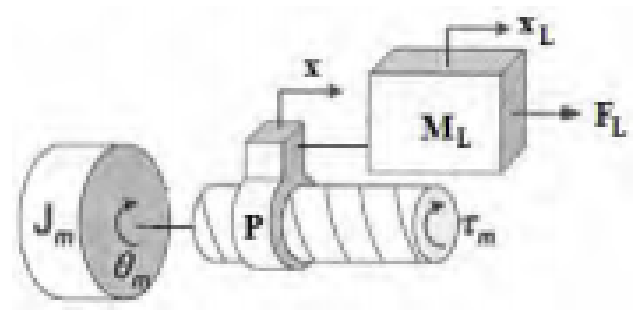

Fig. 1. Lead screw schematic [4]

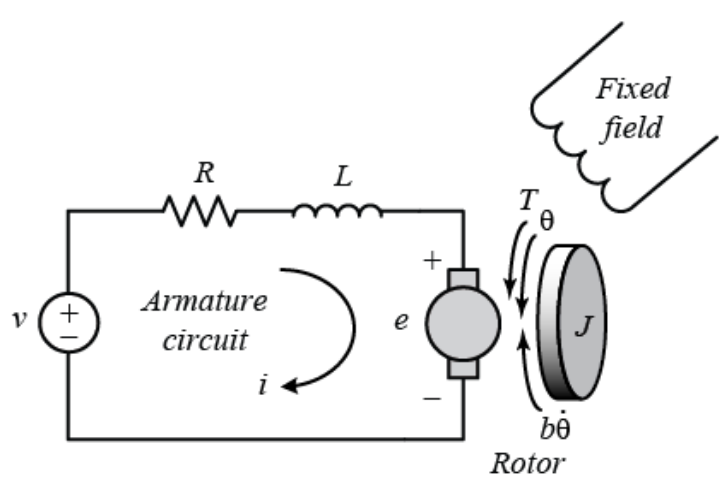

Fig. 2. DC motor electric diagram [5]

\section{A. DC Motor}

Fig. 2 shows the diagram for a DC motor. The torque generated by a DC motor $T_{m}$ is proportional to the armature current $i_{a}$ by a torque constant, $k_{t}$, under the assumption that the magnetic field is constant.

$$
T_{m}=k_{t} i_{a}
$$

The back electromotive force, $e_{b}$, is proportional to the angular velocity of the motor, $\dot{\theta}$ by the fem constant, $k_{b}$.

$$
e_{b}=k_{b} \dot{\theta}
$$

Using Newton's second law and Kirchhoff's voltage law on Fig. 2 and substituting (1) and (2) the governing equations 


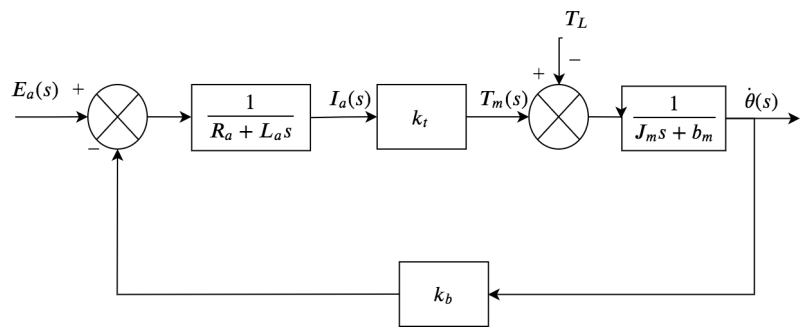

Fig. 3. Block diagram for DC motor model

for the DC motor can be derived.

$$
\begin{gathered}
J_{m} \ddot{\theta}+b_{m} \dot{\theta}=k_{t} i_{a} \\
L_{a} \frac{d i_{a}}{d t}+R_{a} i_{a}=e_{a}-k_{b} \dot{\theta}
\end{gathered}
$$

Where $J_{m}$ is equivalent inertia in the armor, $b_{m}$ is the equivalent viscous damping in the armor, $L_{a}$ is the armature inductance and $R_{a}$ is the armature resistance. $e_{a}$ is the supplied voltage to the motor.

$J_{m}$ and $b_{m}$ are calculated using (5) and (6) to reflect load and damping of the entire load of the lead screw mechanism to the motor.

$$
\begin{gathered}
J_{m}=J_{a}+J_{L}\left(\frac{N_{1}}{N_{2}}\right)^{2} \\
b_{m}=b_{a}+b_{L}\left(\frac{N_{1}}{N_{2}}\right)^{2}
\end{gathered}
$$

Where subscripts $a$ and $L$ represent the armor and load respectively and $N_{1} / N_{2}$ represents the gear ratio between the motor and the load.

By applying the Laplace transformation to (3) and (4), the governing equation can be obtained in the frequency domain.

$$
\begin{gathered}
s\left(J_{m} s+b_{m}\right) \theta(s)=k_{t} I_{a}(s) \\
\left(L_{a} s+R_{a}\right) I_{a}(s)=E_{a}(s)-k_{b} s \theta(s)
\end{gathered}
$$

These equations are the foundations for the plant models of linear position and gripping force [3].

\section{B. Linear Position Model}

The block diagram in Fig is used to demonstrate the model for a DC motor from (7) and (8). The open loop transfer function for a DC motor can be observed by collapsing the block diagram in Fig or by eliminating $I_{a}(s)$ between (7) and (8).

$$
\frac{\dot{\theta}(s)}{E_{a}(s)}=\frac{k_{t}}{\left(J_{m} s+b_{m}\right)\left(L_{a} s+R_{a}\right)+k_{t} k_{b}}
$$

Where the rotational velocity is the output of the plant and the applied voltage is considered the input. The linear position output is desired, as that is the controlled variable. The angular position transfer function can be obtained by integrating (9) and using the relationship between angular position and linear displacement defined in (10).

$$
X(s)=\frac{p}{2 \pi} \theta(s)
$$

Where $p$ is the pitch or lead of the lead screw, $\theta$ is the angular position and $X(s)$ is the linear position [3]. The open loop transfer function for linear position is therefore given as:

$$
\frac{X(s)}{E_{a}(s)}=\frac{k_{t} p}{2 \pi s\left[\left(J_{m} s+b_{m}\right)\left(L_{a} s+R_{a}\right)+k_{t} k_{b}\right]}
$$

\section{Applied Force Model}

In order to obtain the applied force model for the gripping mechanism, the output torque of the motor must be converted to an axial force. For the purposes of this work, the conversion was performed using (12).

$$
T_{m}(s)=\eta p \frac{N_{1}}{N_{2}} F_{g r}(s)
$$

Where $\eta$ is the efficiency and $F_{g r}$ is the resulting linear gripping force [6]. There are more sophisticated methods of converting the motor torque to axial force, however they are more computationally expensive and provide similar results. The resulting open loop linear force transfer function is:

$$
\frac{F_{g r}(s)}{E_{a}(s)}=\frac{k_{t} N_{2}}{\eta p N_{1}\left(L_{a} s+R_{a}\right)}
$$

\section{Control Theory}

\section{A. PD, PI and PID Control}

Proportional-derivative (PD), proportional-integral (PI) and proportional-integral-derivative (PID) controllers are three rudimentary controllers. PD control is used to improve the transient response of a system by feeding the error, as well as the derivative of the error forward to the plant. This results in a very fast response, however leaves the process susceptible to large unwanted signals due to high frequency noise [9]. The standard transfer function for a PD controller is given as

$$
G_{c}(s)=K_{c}+K_{d} s
$$

PI control is commonly used to improve steady state error. Implementing a PI controller increases the system type by one and therefore allows systems to track with no steady state error. Combining an integrated error signal with a proportional gain before inputing to the plant effectively eliminates any bias in the system [9]. The resulting transfer function for a PI controller can be represented as

$$
G_{c}(s)=K_{c}+\frac{K_{i}}{s}
$$

PID control is one of the most common control algorithm used in industry largely due to its robust performance and its ease of implementation. PID controllers utilizes the fast reaction of a PD while improving steady state error with the integral term as with the PI [9]. The corresponding transfer function is

$$
G_{c}(s)=K_{c}+K_{d} s+\frac{K_{i}}{s}
$$




\section{B. Model Predictive Control}

Model predictive controls (MPC) are a method of control that uses a model to make predictions of the future states of the system. The standard MPC algorithm uses the optimization of the control action, $\mathbf{u}$, to minimize the error at futures states. The system model produces a prediction vector, $\hat{\mathbf{y}}$, over a prediction horizon, $N$. The predicted future error is calculated by solving the difference between the prediction and a desired setpoint, $\mathbf{r}$. The control action is optimized given the following cost function:

$$
J=\sum_{j=1}^{N}\left(\hat{y}_{t+j \mid t}-r_{t+j}\right)^{2}+\sum_{j=1}^{n_{u}} \lambda\left(\Delta u_{t+j-1}\right)^{2}
$$

where $\mathbf{r}_{t+j}$ is the reference trajectory at a time instant $j$ steps ahead of the current time instant, $t, \hat{\mathbf{y}}_{t+j \mid t}$ is the predicted output of the system $j$ time steps ahead for the time instant, $t$, and $n_{u}$ is the control horizon, representing the number of future control moves, $\Delta \mathbf{u}$, being considered. In order to ensure a feasible solution to the objection function, a move suppression factor, $\lambda$, is applied to the control moves [7].

A standard MPC that is widely used is known as dynamic matrix control (DMC). This method uses a dynamic matrix A, constructed from an open loop response, a, of the system.

$$
\mathbf{A}=\left(\begin{array}{cccc}
a_{1} & 0 & \cdots & 0 \\
a_{2} & a_{1} & \cdots & 0 \\
\vdots & \vdots & \ddots & \vdots \\
a_{N} & a_{N-1} & \cdots & a_{N-n_{u}}
\end{array}\right)
$$

Optimizing the cost function using least squares method where $\mathbf{E}$ is the current error of the system calculated from (19) and $\mathbf{I}$ is the identity matrix, gives the function (20) for control actions.

$$
\begin{gathered}
\mathbf{E}=\mathbf{r}-\hat{\mathbf{y}} \\
\boldsymbol{\Delta} \mathbf{u}=\left(\mathbf{A}^{T} \mathbf{A}+\lambda \mathbf{I}\right)^{-1} \mathbf{A}^{T} \mathbf{E}
\end{gathered}
$$

The prediction is then calculated using $\Delta u$. The expression for $\hat{y}$ is:

$$
\left.\hat{y}\right|_{t}=\left.\hat{y}\right|_{t+1}+\mathbf{A} \boldsymbol{\Delta} u+\left.\phi\right|_{t}
$$

where $\phi$ is a correction factor calculated from the difference between the measured value of the plant output, $y_{m}$, and the model prediction.

$$
\left.\phi\right|_{t}=y_{m}-\left.\hat{y}\right|_{t-1}
$$

The described algorithm for MPC derived from the work of [7]. DMC is suitable for tracking setpoints for asymptotically stable linear systems [8], thus for unstable systems such as the one described in this work, a stabilizing technique must be executed in order for a DMC to be implemented.

\section{Cascading MPC-PD and MPC-PI}

The method of stabilization for DMC implementation in this application was to cascade a PD/PID. These cascaded controllers create PD based and PI based MPC (MPC-PD and MPC-PI respectively). The DMC model in these variations becomes a closed loop PD/PI controlled system as opposed to the usual plant model. The block diagram in FIG demonstrates the cascading control loop. The DMC is effectively providing the setpoint for the PD/PI controllers.

\section{Preliminary Simulations and Results}

Preliminary simulations were performed to analyze the performance of the described control schemes on the individual plant models using the system parameters in Table I and the control parameters summarized in Table II. The control parameters were chosen using MATLAB2019b PID tuning tools. First, closed loop gripping force simulations were performed using the PD, PID and MPC-PD control schemes. An arbitrary setpoint was chosen of $0.1 \mathrm{~m}$, which is approximately $60 \%$ of the maximum travel of the lead screw.

TABLE I

SYSTEM PARAMETERS

\begin{tabular}{lll}
\hline Model Parameter & Value & Units \\
\hline Equivalent inertia, $J_{m}$ & 0.0043 & $\mathrm{~kg} / \mathrm{m}^{2}$ \\
Equivalent viscous damping, $b_{m}$ & 0.0038 & $\mathrm{Ns} / \mathrm{m}$ \\
Gear ratio, $\frac{N_{1}}{N_{2}}$ & 4.4 & \\
Armature resistance, $R_{a}$ & 2.32 & $\Omega$ \\
Armature inductance, $L_{a}$ & 0.238 & $\mathrm{mH}$ \\
Pitch, $p$ & 7.5 & $\mathrm{~mm}$ \\
Torque constant, $k_{t}$ & 21.3 & $\mathrm{mNm} / \mathrm{A}$ \\
fem constant, $k_{b}$ & 0.0024 & $1 / \mathrm{sV}$ \\
Efficiency, $\eta$ & 20 & $\%$ \\
Max travel length, $L_{l s}$ & 0.150 & $\mathrm{~m}$ \\
Gravitational constant, $g$ & 9.81 & $\mathrm{~m} / \mathrm{s}^{2}$ \\
\hline
\end{tabular}

The preliminary results for position control are demonstrated in Fig. 5 and a qualitative analysis was performed in order to determine the preeminent controller which will be implemented in the total process control scheme. The analysis included comparison of the root-mean-square (RMS) error, the settling time and the percentage overshoot. From Table III it was concluded that the MPC-PD outperformed both the PID and PD in all three metrics. This is likely due to the MPC-PD's ability to limit move aggressiveness, leading to smoother response with less overshoot than the PD control. The increased response time associated with the PID control makes it less desirable for implementation as robotic gripping is a fast process.

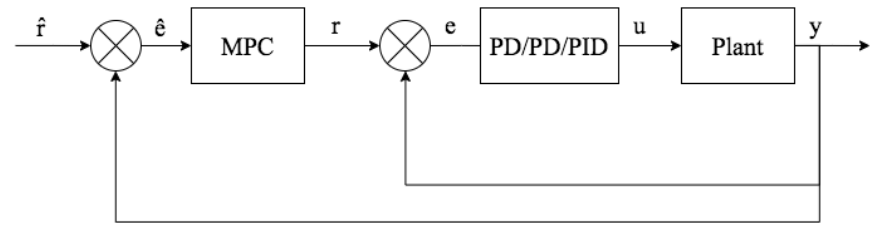

Fig. 4. Cascading control loop 
TABLE II

CONTROL PARAMETERS

\begin{tabular}{llll}
\hline System & Controller & Parameter & Value \\
\hline & PD & $k_{c}$ & 968 \\
& & $k_{d}$ & 364 \\
Position & PID & $k_{c}$ & 653 \\
$t_{s}=0.01$ & & $k_{i}$ & 148 \\
& & $k_{d}$ & 640 \\
& MPC-PD & $k_{c}$ & 968 \\
& & $k_{d}$ & 364 \\
& & $N$ & 700 \\
& & $n_{u}$ & 10 \\
Force & & $\lambda$ & 1.001 \\
$t_{s}=0.0001$ & & $k_{c}$ & 18183 \\
& & $k_{i}$ & 1.0291 \\
& & $k_{c}$ & 18183 \\
& & $k_{i}$ & 1.0291 \\
& & $N$ & 100 \\
& & $n_{u}$ & 10 \\
& & $\lambda$ & 1.005 \\
\hline
\end{tabular}

Subsequently, two control schemes were implemented on the force feedback system in simulation: a PI control and a cascaded MPC-PI. The results in Fig. 6 show the simulation attempted to control the system for a setpoint of $1 \mathrm{~N}$. DC motor torque is fast system and therefore simulation time was short and required small sampling instances. The analysis of performance by the same three metrics as above is summarized in Table IV. The MPC-PI resulted in a lower RMS error, however it introduces a steady-state error. In addition, due to the natural speed of the force response reaching steady state, it would be difficult to implement the MPC-PI in real world application as the computational time would most likely exceed the necessary sampling time.

TABLE III

RMS ERROR FOR POSITION

\begin{tabular}{llll}
\hline Controller & PD & PID & MPC-PD \\
\hline RMS Error & 0.0242 & 0.0390 & 0.0238 \\
Overshoot & 7.2985 & 0.0469 & 4.7207 \\
Settling Time (s) & 4.6404 & 10.8165 & 3.1805 \\
\hline
\end{tabular}

TABLE IV

RMS ERROR FOR FORCE

\begin{tabular}{lll}
\hline Controller & PI & MPC-PI \\
\hline RMS Error & 0.0888 & 0.0360 \\
Overshoot & 2.4345 & 8.8266 \\
Settling Time (s) & $3.557 \mathrm{e}-04$ & 0.0011 \\
\hline
\end{tabular}

\section{CONTROLler IMPlementation}

The desired outcome of this paper is to control both the position of the gripper finger and the amount of force applied to an object being grasped. This requires separate control schemes for each and a switching mechanism that recognizes when the fingers have made contact with an object and subsequently maintain a desired gripping force and therefore a desired motor torque. The entire control block diagram is shown in Fig. 7. The position stage is to be regulated by
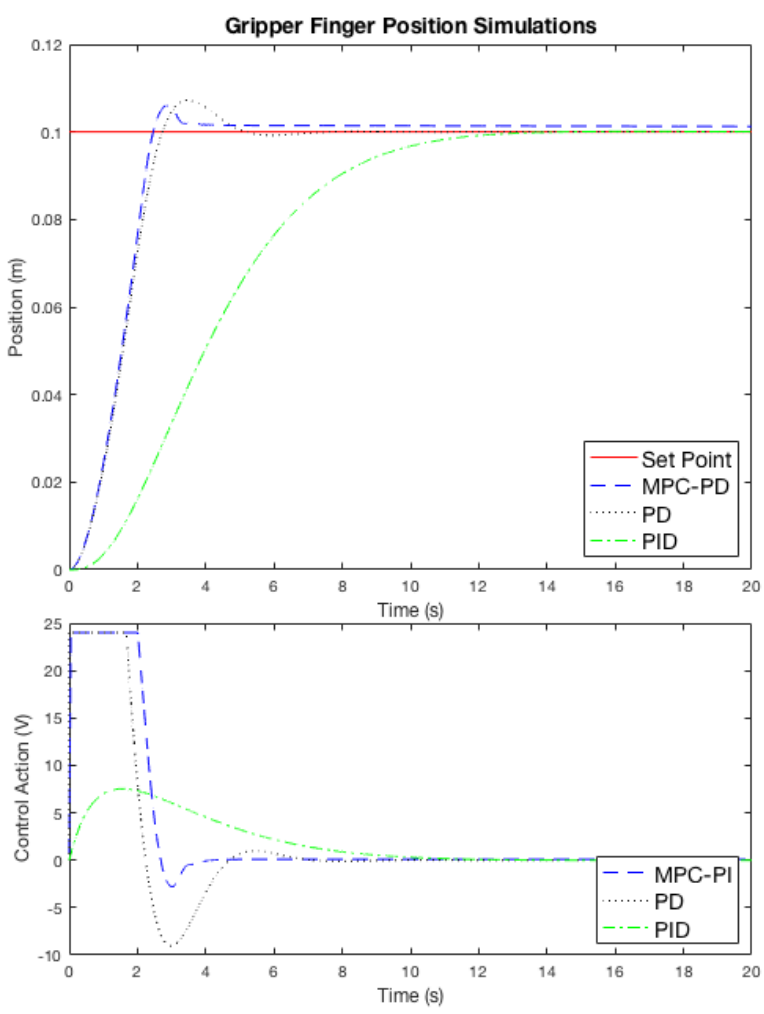

Fig. 5. Simulation results for gripper position

MPC-PD control and the force stage is regulated by PI control. Before the gripping mechanism control begins, setpoints for position and force are determined based on the information given to the controller about the size, weight and position of the object in the workspace.

\section{A. Position Feedback Control}

The initial stage of the controller is the position feedback control. The position setpoint is calculated as the difference between the width of the object and the travel length of the lead screw, $\left(L_{l s}\right)$. In the implementation of the MPC-PD, the error between the plant position and the setpoint is the input for the DMC controller.the DMC determines the setpoint for the PD controller. The PD controller then determines the control action to provide the plant.

\section{B. Switching Criterion}

There are two switching criterion that will be checked at each time instant. If either condition is true, the position control stage ends and the force feedback control stage begins. The first condition verifies whether the position setpoint has been reached within a tolerance of $\pm 2 \%$. If this condition has not been met, the controller checks the second condition. The second condition reads force sensors on the gripper fingers and determines whether there has been a change in the voltage reading above a set tolerance. This tolerance will be the width 

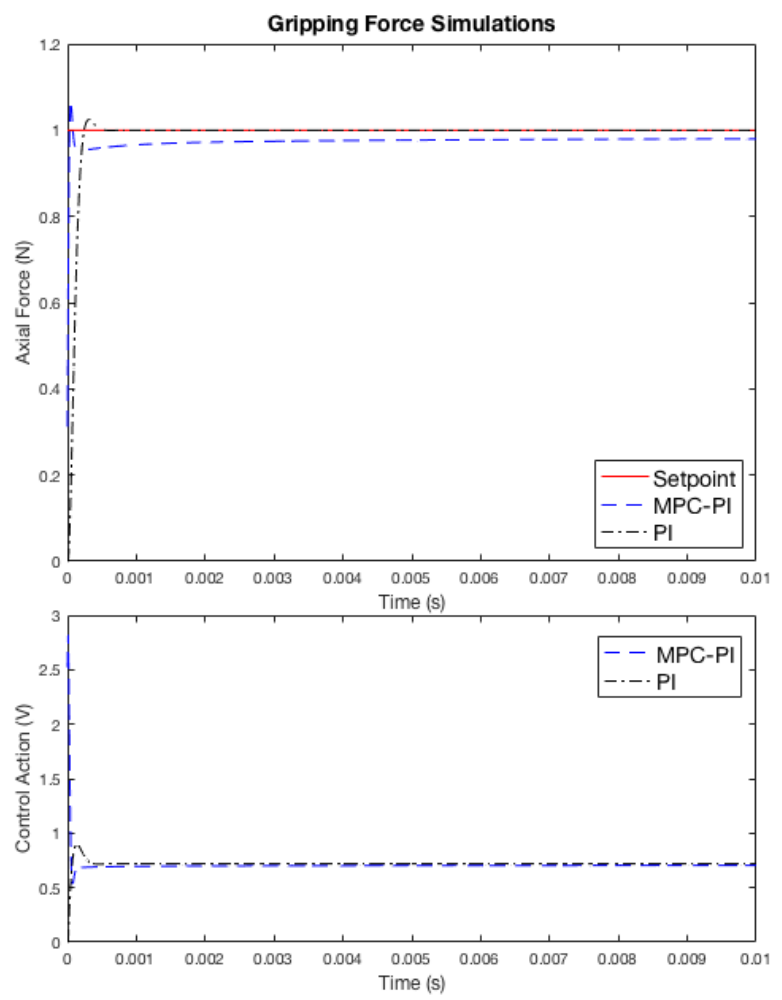

Fig. 6. Simulation results for gripping force

of voltage range required to activate the sensors. If neither condition is true, the position controller will continue to iterate through another time step. Since this work examines only the simulation of the system and the force sensors cannot be easily implemented in simulation, the position setpoint condition will always be the switching criterion.

\section{Force Feedback Control}

The second stage of the controller is the force feedback control. When either switching criterion is met, the force setpoint, $\left(F_{s p}\right)$ calculated as $(23)$ is initialized and gripping force control begins.

$$
F_{s p}=\mu_{s} m(g+a)
$$

Where, $F_{s p}$ is variable depending on the acceleration of the object, $a$, for this application $a=0$, as it is assumed the gripper is not attached to a manipulator. $\mu_{s}$ is defined as the static coefficient of friction between the object surface and the material of the gripper fingers, $m$ is the mass of the object and $g$ is acceleration due to gravity. The PI is then implemented using difference between $F_{s p}$ and $F_{g r}$ as the error inputed into the controller. The PI controller subsequently determines the appropriate control action to provide the plant. During this stage of control, the position of the gripper finger is still being observed, to ensure contact between the object and the fingers are not lost. The position should remain constant duing the force control stage, as the sum of the forces between the object and the fingers should be zero. The forces when reflected back to the motor as torques $\left(T_{m}\right.$ and $\left.T_{L}\right)$ will also sum to zero. From Fig. 7 is should be noted that the signal entering the inertia and damping block is this sum of torques, causing the velocity and hence the change in position to be zero as well.

\section{Simulation Results}

The complete process control was implemented in simulation on three different objects. The relevant characteristics of these objects are summarized in Table V. The results in Fig. 8 demonstrate the position, force an control action through the postion control stage, the switch criterion and the force control stage of the process. In all three cases, the gripper was able to reach the correct position setpoint before switching to force control. Once the switching criterion is met, the process is also able to accurately track the desired gripping force rather quickly. Limits were set for the control action, as the DC motor has a nominal voltage of $24 \mathrm{~V}$ that should not be exceeded. The applied forces generated before the fingers experience contact with object although not important during the position regulation stage, cause overshoot as the process switches to the force control. The applied forces during the first few instances of contact are up to three times the desired gripping force. This is an issue that will need to be addressed in future work when translated to real world applications. When dealing with robust objects in practice, this spike in applied force may not cause issues, however, a spike in applied force to a more fragile or brittle object may be disastrous.

\section{TABLE V}

\begin{tabular}{l|ll}
\hline & Width (m) & Mass (kg) \\
\hline Object 1 & 0.01 & 1.27 \\
Object 2 & 0.05 & 1.69 \\
Object 3 & 0.10 & 0.75 \\
\hline
\end{tabular}

\section{CONClusions ANd Future Work}

This research laid the ground work for developing a controller for a flexible two-finger parallel electric gripper. The objective was to develop a control scheme that could regulate both the position and applied force of the gripper. This objective was met with the development of a switching MPC$\mathrm{PD} / \mathrm{PI}$ control scheme. The solution outlined above combines the DMC's optimization of the control move each time step with the stabilizing ability of the PD to provide efficient position control, while subsequently providing a fast transition to gripping force control through a switching condition. Future work includes the implementation of the control process on an experimental setup. The experimental setup will consist of the electric gripper fastened to the end of a robotic manipulator as could be found in industrial pick and place applications. Modifications will be made to address the issues with the spike in force and the nonlinearity associated with flexible gripper fingers. An improved system model will be developed through system identification techniques. 


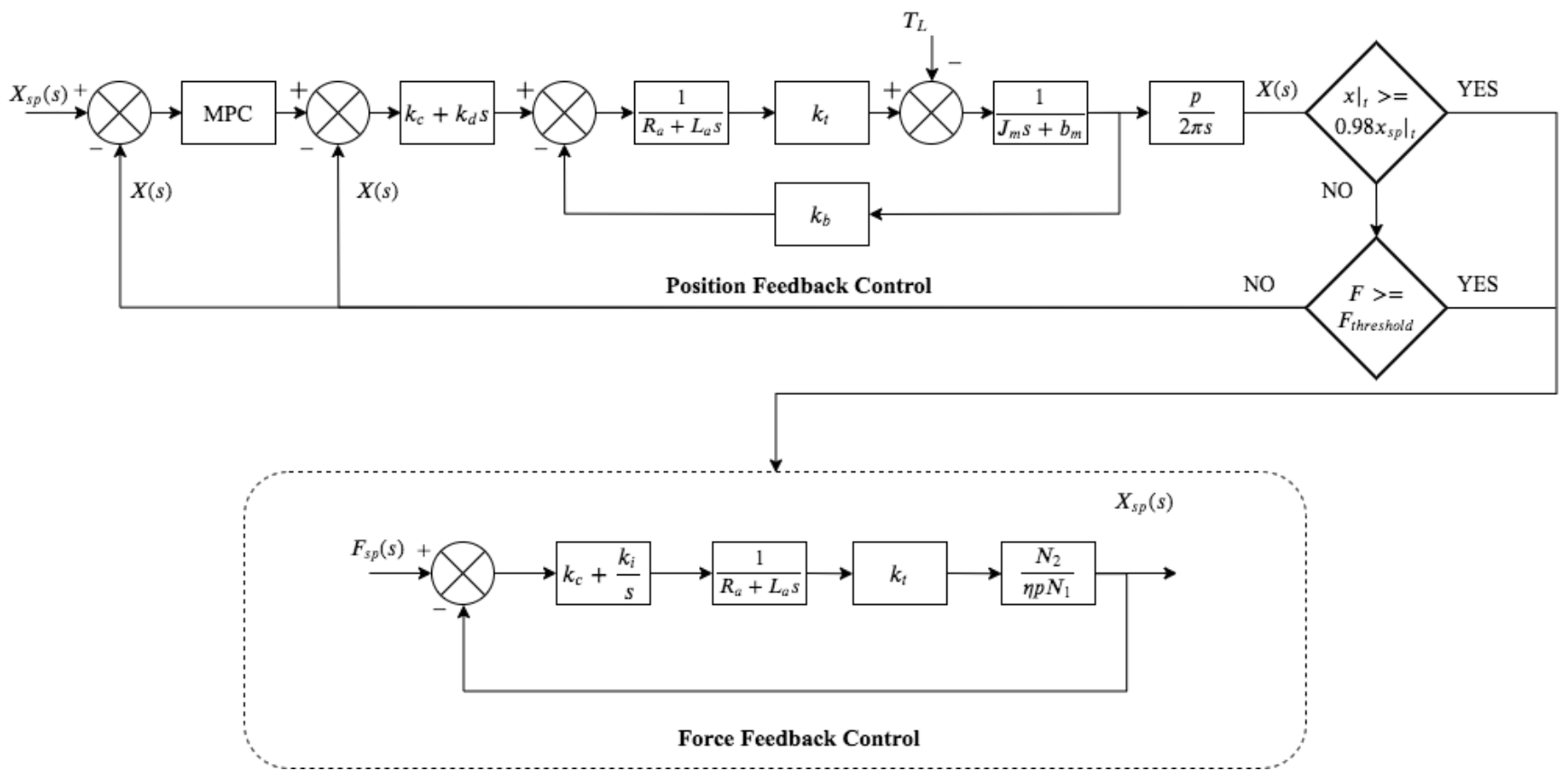

Fig. 7. Block diagram of complete control process
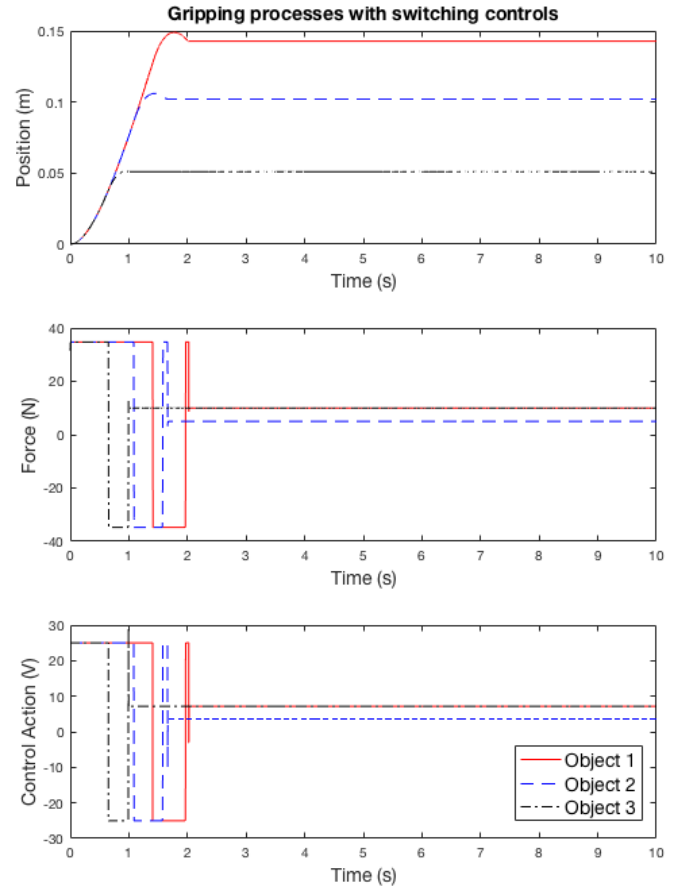

Fig. 8. Object characteristics

\section{REFERENCES}

[1] S. Sundaram, P. Kellnhofer, Y. Li, J.-Y. Zhu, A. Torralba, and W. Matusik, "Learning the signatures of the human grasp using a scalable tactile glove," Nature, vol. 569, no. 7758, pp. 698-702, May 2019, doi: 10.1038/s41586-019-1234-z.
[2] C. Chen and C. Lan, "An Accurate Force Regulation Mechanism for High-Speed Handling of Fragile Objects Using Pneumatic Grippers," IEEE Transactions on Automation Science and Engineering, vol. 15, no. 4, pp. 1600-1608, Oct. 2018, doi: 10.1109/TASE.2017.2757527.

[3] B. Hannaford and A. M. Okamura, "Haptics," in Springer Handbook of Robotics, B. Siciliano and O. Khatib, Eds. Berlin, Heidelberg: Springer Berlin Heidelberg, 2008, pp. 719-739.

[4] "Control Tutorials for MATLAB and Simulink - Motor Position: System Modeling." [Online]. [Accessed: 02-Feb-2020].

[5] E. D. Ruiz-Rojas, J. L. Vazquez-Gonzalez, R. Alejos-Palomares, A. Z. Escudero-Uribe, and J. R. Mendoza-Vázquez, "Mathematical Model of a Linear Electric Actuator with Prosthesis Applications," in 18th International Conference on Electronics, Communications and Computers (conielecomp 2008), Puebla, Mexico, 2008, pp. 182-186, doi: 10.1109/CONIELECOMP.2008.29.

[6] J. R. Derges, "Torque control of a separate excitation DC motor for a dynamometer," p. 78.

[7] . Wilson, M. Charest, and R. Dubay, "Non-linear model predictive control schemes with application on a 2 link vertical robot manipulator," Robotics and Computer-Integrated Manufacturing, vol. 41, pp. 23-30, Oct. 2016, doi: 10.1016/j.rcim.2016.02.003.

[8] Y. Xi and D. Li, Predictive Control: Fundamentals and Developments. John Wiley Sons, 2019.

[9] N. S. Nise, Control systems engineering, Seventh edition. Hoboken, NJ: Wiley, 2015. 